\title{
Recomendaciones para la reactivación de laboratorios de pruebas de función pulmonar durante la pandemia por SARS-CoV-2 (COVID-19) en Colombia
}

\author{
Recommendations for the reactivation of laboratories \\ of lung function tests during the pandemic by \\ SARS-CoV-2 (COVID-19) in Colombia
}

\author{
María José Fernández Sánchez, MD ${ }^{1,2}$, Rafael Acero Colmenares, MD ${ }^{1,3}$, Carlos Eduardo \\ Aguirre Franco, MD ${ }^{1,4}$, Alejandro Casas Herrera, MD ${ }^{1,4}$, Diego Miguel Celis Mejía, MD ${ }^{1,5}$, Clara \\ Patricia García Calderón, MD ${ }^{1,6}$, Mauricio González García, MD ${ }^{1,4}$, EIDELMAN ANTONIO González, \\ MD ${ }^{1,7}$, Gustavo Adolfo Hincapié, MD ${ }^{1,8}$, JaVier IVÁn LAsso, MD ${ }^{1,2}$, Carlos Eduardo Matiz Bueno,

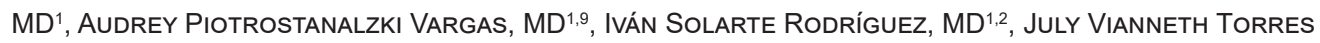 \\ GONZÁLEZ, MD ${ }^{1,10}$, JUAN DAVID BOTERo BAHAMON, MD ${ }^{1,2}$
}

\section{Introducción}

La pandemia de COVID-19 plantea retos y desafíos para la salud pública y para la provisión de servicios de salud en el mundo, y Colombia no es la excepción. Con la evidencia disponible hasta el momento, se sabe que el mecanismo de transmisión más probable del SARS-CoV-2 es a distancia por gotas respiratorias mayores de 5 micras (gotas de Flügge), que no permanecen suspendidas en el aire y que se depositan en las superficies a menos de 2 metros, por contacto de las secreciones infectadas directamente con las mucosas (oral, nasal, conjuntiva) o indirectamente (a través de las manos contaminadas). No se excluye la trasmisión por aerosoles, partículas de diámetro menor de 5 micras, generadas por la tos y por procedimientos invasivos, como la toma de muestras, intubación o broncoscopia, y durante las maniobras para las pruebas de función pulmonar (PFP), razón por la cual, estas representan un alto riesgo de contagio en los laboratorios de función pulmonar.

Ante este escenario se deben optar por medidas preventivas, para lo cual es necesario hacer las mejores recomendaciones, que eviten el contagio durante las pruebas, adoptando precauciones de seguridad adicionales a las ya existentes, durante y después de su realización, que inevitablemente conducirán a tiempos más largos de espera y mayor duración de las pruebas, incremento en la utilización de insumos desechables, menor flujo de pacientes y aumento de los costos. Para la reactivación de los laboratorios de función pulmonar se deben tener en cuenta la etapa de la pandemia, la urgencia del procedimiento, según el estado clínico y diagnóstico del paciente, y el tipo de prueba (Tabla 1 y 2 ).

\section{Recomendaciones para la fase de pandemia (alta prevalencia de COVID-19 en la comunidad)}

Durante el período de alta prevalencia del virus en la comunidad se debe considerar cuidadosamente la seguridad del personal, la contaminación cruzada del equipo y, por tanto, restringir las PFP a pacientes que requieren pruebas para tomar decisiones urgentes/esenciales para el diagnóstico o el tratamiento inmediato de la enfermedad.
'Médico Internista y Neumólogo, Comité de Función Pulmonar, Asociación Colombiana de Neumología y Cirugía de Tórax

${ }^{2}$ Facultad de Medicina, Pontificia Universidad Javeriana; Unidad de Neumología, Hospital Universitario San Ignacio.

${ }^{3}$ Facultad de Medicina, Universidad de los Andes; Hospital Universitario, Fundación

Santafé de Bogotá.

${ }^{4}$ Fundación Neumológica Colombiana.

${ }^{5}$ Facultad de Medicina, Universidad Pontificia Bolivariana; servicio de Neumología

Clínica CardioVID.

${ }^{6}$ Facultad de Medicina, Universidad Pontificia Bolivariana; Unidad de Neumología, Hospital Pablo Tobón Uribe; Laboratorio de función pulmonar, Fundación Antioqueña de Infectología.

${ }^{7}$ Facultad de Medicina, Universidad Metropolitana de Barranquilla; IPS Centro de Atención Pulmonar CAP Ltda.

${ }^{8}$ Facultad de Medicina, Universidad Militar Nueva Granada; Servicio de Neumología, Hospital Militar Central.

${ }^{9}$ IDIME, Armenia.

${ }^{10}$ Servicio de Neumología, Hospital Universitario Nacional de Colombia; Clínicos IPS.

Autor de correspondencia:

María José Fernández Sánchez.

Correo electrónico: m.fernandez@javeriana.edu.co

Recibido: $15 / 05 / 2020$

Aceptado: 30/05/2020 
Tabla 1. Clasificación del riesgo de las pruebas de función pulmonar

Procedimientos generadores de aerosoles

\begin{tabular}{|ll}
\multicolumn{1}{c}{ Alto riesgo } & \multicolumn{1}{c}{ Riesgo medio } \\
- Prueba de ejercicio & - Espirometría pre y \\
cardiopulmonar & posbroncodilatador \\
(ergoespirometría, & Capacidad de difusión \\
caminata de 6 minutos, & de monóxido de carbono \\
shuttle test) & (DLCO) \\
- Nebulización de & Volúmenes pulmonares \\
medicamentos, por & por pletismografía \\
ejemplo, metacolina & Resistencias por \\
Otras pruebas de & oscilometría forzada \\
provocación bronquial, & - Medición de presiones, \\
como ejercicio & PIM y PEM \\
& - FeNO \\
& - Medición de pico/flujo \\
& - Lavado de nitrógeno \\
& - Gases arteriales \\
\hline
\end{tabular}

FeNO: fracción exhalada de óxido nítrico; PEM: presión máxima espiratoria; PIM: presión máxima inspiratoria.

Tabla 2. Pruebas de función pulmonar sugeridas de acuerdo con la etapa de la pandemia

\begin{tabular}{|c|c|}
\hline Durante la pandemia & Después de la pandemia \\
\hline $\begin{array}{l}\text { - Espirometría y DLCO } \\
\text { - Considerar los } \\
\text { volúmenes pulmonares } \\
\text { por pletismografía y la } \\
\text { medición de resistencias } \\
\text { de la vía aérea por } \\
\text { oscilometría forzada } \\
\text { - Medición de presiones, } \\
\text { PIM y PEM } \\
\text { - Medición de pico/flujo }\end{array}$ & $\begin{array}{l}\text { Prueba de ejercicio } \\
\text { cardiopulmonar } \\
\text { (ergoespirometría, } \\
\text { caminata de } 6 \text { minutos, } \\
\text { shuttle test) } \\
\text { - Pruebas de provocación } \\
\text { bronquial }\end{array}$ \\
\hline
\end{tabular}

En cualquier circunstancia, la realización de PFP está contraindicada en pacientes con sospecha clínica de infección respiratoria aguda de cualquier tipo. Adicionalmente, en esta etapa de la pandemia no se deben hacer pruebas de función pulmonar a los pacientes con sospecha o diagnóstico de infección por SARS-CoV-2, entendiendo que en el momento actual de transmisión comunitaria y de desabastecimiento de equipos de protección individual (EPI), cualquier sintomatología respiratoria de reciente aparición se presupone asociada con COVID-19 (aún con una prueba de PCR negativa).
Se deben diferir las pruebas de función pulmonar que no sean imprescindibles (Tabla 3). Los pacientes con COVID-19 no deben ser evaluados durante un mínimo de 30 días después de la infección.

Tabla 3. Indicaciones de pruebas de función pulmonar durante la pandemia

Indicaciones para hacer pruebas de función pulmonar durante la pandemia

- Preoperatorio de cirugía de resección pulmonar, en especial, si es oncológica

- Preoperatorio de cirugía cardíaca

- Para diagnóstico o seguimiento de enfermedades intersticiales difusas, cuyo resultado implique necesariamente una modificación del tratamiento

- Para el seguimiento del trasplante pulmonar o durante la valoración pretrasplante

- Para el diagnóstico de enfermedades pulmonares que no respondan a tratamientos empíricos

Pruebas de función pulmonar contraindicadas durante la pandemia

Las pruebas de ejercicio, nebulizaciones, provocación bronquial y otras generadoras de aerosoles deben posponerse. Los pacientes con situaciones especiales se deberán valorar en forma individualizada, sopesando la relación riesgo-beneficio

\section{Organización de los servicios}

\section{Recomendaciones antes de hacer las pruebas}

\section{Recomendaciones que se deben dar durante la asignación de la cita}

- Confirme con el paciente si, de acuerdo con las indicaciones de su médico tratante, la prueba es esencial para el manejo de su caso.

- En todos los casos, sin excepción, se debe preguntar al paciente si ha tenido contacto con alguna persona diagnosticada con COVID-19 o si ha tenido síntomas relacionados, como fiebre o escalofríos, malestar general (cefalea, mialgias y artralgias), dolor de garganta, tos, alteraciones del gusto y olfato o alguna otra molestia respiratoria (Tabla 4).

- En caso afirmativo, indicar al paciente que debe comunicarse a la línea de atención telefónica 123 o con su EPS, y reprogramar la cita en un lapso no menor de 20 días. 
Tabla 4. Lista de chequeo para pruebas de función pulmonar

\begin{tabular}{|c|c|c|c|c|c|c|}
\hline \multirow[b]{2}{*}{ 1. ¿Se ha realizado pruebas para COVID-19? } & \multicolumn{2}{|c|}{ Día de solicitud de cita } & \multicolumn{2}{|c|}{72 horas antes } & \multicolumn{2}{|c|}{ Día de la cita } \\
\hline & Sí & No & $\mathrm{Si}$ & No & Sí & No \\
\hline $\begin{array}{l}\text { 2. Tipo de prueba: } \\
\text { (llenar en caso de tener prueba) }\end{array}$ & Sangre & Hisopo & Sangre & Hisopo & Sangre & Hisopo \\
\hline $\begin{array}{l}\text { 3. Resultado de prueba: (llenar en caso de tener } \\
\text { prueba) }\end{array}$ & Positivo & Negativo & Positivo & Negativo & Positivo & Negativo \\
\hline 4. Fiebre $37,5^{\circ} \mathrm{C}$ o más & Sí & No & Sí & No & Sí & No \\
\hline 5. Malestar general & Sí & No & Sí & No & Sí & No \\
\hline 6. Dificultad para respirar & Sí & No & Sí & No & Sí & No \\
\hline 7. Dolor de pecho & Sí & No & Sí & No & Sí & No \\
\hline 8. Dolor de garganta & Sí & No & Sí & No & Sí & No \\
\hline 9. Pérdida del sentido del olfato & Sí & No & Sí & No & Sí & No \\
\hline 10. Pérdida del sentido del gusto & Sí & No & Sí & No & Sí & No \\
\hline 11. Tos & Sí & No & Sí & No & Sí & No \\
\hline 12. Náuseas & Sí & No & Sí & No & Sí & No \\
\hline 13. Conjuntivitis & Sí & No & Sí & No & Sí & No \\
\hline 14. Vómito & Sí & No & Sí & No & Sí & No \\
\hline 15. Diarrea & Sí & No & Sí & No & Sí & No \\
\hline 16. Flujo nasal & Sí & No & Sí & No & Sí & No \\
\hline 17. ¿Ha viajado recientemente fuera de la ciudad? & Sí & No & Sí & No & Sí & No \\
\hline $\begin{array}{l}\text { 18. En los últimos } 14 \text { días, ¿ha entrado en } \\
\text { contacto cercano (a menos de } 2 \text { metros) con } \\
\text { alguien que tiene diagnóstico de COVID-19 } \\
\text { confirmado por laboratorio? }\end{array}$ & Sí & No & Sí & No & Sí & No \\
\hline 19. ¿Es trabajador de la salud? & Sí & No & Sí & No & Sí & No \\
\hline 20. ¿Es cuidador de persona adulta? & Sí & No & Sí & No & Sí & No \\
\hline
\end{tabular}

- Solo si el paciente es menor de edad o dependiente de cuidador, este podrá asistir acompañado de una única persona, quien, a su vez, no deberá haber tenido contacto con alguna persona diagnosticada con COVID-19 o síntomas relacionados.

- Recuerde al paciente que debe usar tapabocas o máscara, y cuando ingrese al hospital y al servicio debe higienizar sus manos con alcohol glicerinado o, en el mejor de los casos, deberá lavar sus manos con agua y jabón.

\section{Pruebas según la indicación y prioridad}

- Las PFP deben limitarse a la espirometría, difusión de monóxido de carbono, volúmenes pulmonares por pletismografía, resistencias de la vía aérea por oscilometría forzada, PIM y PEM.

- Para otras pruebas, el neumólogo a cargo del laboratorio debe evaluar el riesgo y la indicación.

\section{Recomendaciones de infraestructura}

- Reorganice las áreas de espera, los pacientes deben usar tapabocas o máscara, y deben sentarse separados por una distancia mínima de 2 metros.

- Reorganice la sala de pruebas y las oficinas del personal para minimizar la transmisión del virus. Idealmente, designar áreas de pruebas separadas para pacientes hospitalizados y ambulatorios. 
- Reorganice los horarios. Asigne el tiempo para cada prueba, que incluya el tiempo necesario para la limpieza y descontaminación de equipos y superficies entre cada prueba.

- Permita al menos 15 minutos de ventilación de la sala de pruebas (las ventanas abiertas con las puertas cerradas, mejor si tiene disponible presión negativa) y el tiempo necesario para que el personal se ponga sus elementos de protección personal (EPP).

- Se debe calibrar el equipo de función pulmonar después de la descontaminación (aplica para los equipos sin filtro con sensor expuesto, en especial, neumotacógrafos de turbina).

- El tiempo extra requerido será de 30 a 60 minutos

- Debe contar con personal para brindar apoyo y supervisión al técnico de pruebas en el uso de los EPP (puesta y retirada).

\section{Recomendaciones para la realización de las pruebas}

\section{Adecuación de la infraestructura donde se hacen las pruebas de función pulmonar}

- Las superficies de trabajo deben estar libres de todo material. El teléfono, el computador y los documentos necesarios deben estar en un lugar distante al equipo, de al menos 1 metro e idealmente 2 metros.

- El paciente se orienta de tal forma que la exhalación no se dirija hacia el lugar que ocupa el técnico.

- El espacio y la disposición del equipo deben permitir que la distancia entre el paciente y el técnico sea al menos de 2 metros, y el técnico debe poder ubicarse detrás y al lado del paciente.

- Cada sala debe disponer de pañuelos de papel para el paciente y contenedores para residuos con tapa de pedal.

- Cada sala de examen debe disponer de dispensadores de gel antibacterial, alcohol o toallas húmedas desinfectantes para la higiene de manos del personal y pacientes.

- Es necesario que el paciente use su tapabocas o máscara antes y después del examen.
- Se debe decidir por el médico del laboratorio si es necesario el uso de una prueba posB2. En caso de requerirlo, este inhalador debe ser personal, para uso individual por paciente.

\section{Equipos empleados para realizar las PFP}

- Las pruebas siempre deben realizarse con filtros desechables para protección bacteriana y viral de alta especificidad. Filtros con eficiencia mínima comprobada para flujos espiratorios de 600 a $700 \mathrm{~L} / \mathrm{min}$. No se recomienda el uso solo de boquilla/sensor combinados. La excepción sería cuando se pueda agregar un filtro a la boquilla/sensor (entre el paciente y la boquilla).

- Prefiera el uso de insumos desechables, deseche los artículos con cuidado, por ejemplo, pinzas para la nariz, boquillas de goma. En el caso que sean reutilizables deberán manejarse como riesgo biológico, para ser llevados a la central de esterilización.

- Si durante la prueba se utilizan insumos como mangueras corrugadas, bolsas, espaciadores o válvulas, estos siempre deben cambiarse entre pacientes.

- Los pacientes con alto riesgo de infección por SARS$\mathrm{CoV}-2$, que necesariamente requieren pruebas, deberían estar en una sala con presión negativa y equipos destinados únicamente para este grupo de enfermos. Dado que estas condiciones solo están disponibles en centros especializados, como alternativa se plantea el uso de una sala con buena ventilación ambiental (ventanas abiertas con puerta cerrada).

\section{Limpieza y control de infecciones}

\section{Personal asistencial}

El equipo de protección personal es obligatorio en todas las circunstancias. El uso de EPP en áreas de alto riesgo debe gestionarse dentro del mismo espacio. El personal no puede salir del área donde se hace la prueba sin quitarse el EPP según el protocolo institucional.

\section{Protocolos de limpieza de las instalaciones y de los equipos}

- Una vez que el paciente ha salido de la sala, se procederá a la limpieza de todas las superficies con toallitas 
desechables empapadas con el antiséptico aprobado por su institución, en general, amonio cuaternario, alcohol etílico al $60 \%$ o $70 \%$, peróxido de hidrógeno al $0,5 \%$ o solución de agua e hipoclorito de sodio. La limpieza y desinfección de paredes y pisos se hará conforme al procedimiento habitual de cada centro.

- Se hará limpieza de todos los componentes que entran en contacto con el paciente, incluido el mango del cabezote o pieza de boca, líneas del neumotacógrafo, silla, cabina, entre otros.

- Los protocolos regulares de limpieza y mantenimiento de equipos deben revisarse y aplicarse localmente de acuerdo con las recomendaciones del fabricante y en estricto cumplimiento.

- La sala de pruebas debe tener la mejor ventilación, idealmente recambio de aire con presión negativa en sitios donde esté disponible. No se recomiendan los filtros HEPA, porque se contaminan con gérmenes y partículas virales. En caso de contar con ellos se recomienda su cambio periódico.

- Donde sea posible, se puede hacer descontaminación de la sala con vaporización de ozono o con emisión de luz UV a intervalos, según la política local de control de infecciones.

- Implemente un estricto control de cumplimiento de los protocolos de limpieza para el control de infecciones en su institución.

\section{Recomendaciones para la fase de baja prevalencia de COVID-19 en la comunidad}

En esta fase de baja prevalencia de la enfermedad, la probabilidad de infección es menor que en la fase pandémica, sin embargo, se requieren las mismas medidas de seguridad. Por tanto, los procedimientos esenciales de control de infección y las recomendaciones organizativas deben continuar como en la fase de gran contagio.

\section{Pruebas y equipamiento}

Se pueden hacer todas las PFP con las precauciones de seguridad, como son el uso de EPP completo (gafas de protección, máscara N95 o superior, careta y bata antifluido para el caso de las pruebas de ejercicio cardiopulmonar) (Tabla 5).

El uso de filtros conectados al puerto de inhalación/ exhalación de la mascarilla o boquilla durante las pruebas de ejercicio puede reducir la transmisión de aerosoles. Tenga en cuenta que esto puede resultar en un aumento de resistencia al flujo de aire, a medida que aumenta la demanda ventilatoria de ejercicio (humidificación del filtro y el incremento de la resistencia al aumento del flujo ventilatorio), lo que hace los resultados de ejercicio menos confiables, especialmente en pacientes con disnea; aún no hay un consenso sobre su uso. En este momento no podemos recomendar el uso de filtros durante las pruebas de ejercicio y se deben usar EPP completo al realizar estas pruebas.

Tabla 5. Elementos de protección personal según nivel de riesgo de las pruebas de función pulmonar

\begin{tabular}{|lcc|}
\hline \multicolumn{1}{|c}{ EPP } & $\begin{array}{c}\text { Alto } \\
\text { riesgo }\end{array}$ & $\begin{array}{c}\text { Riesgo } \\
\text { medio }\end{array}$ \\
\hline $\begin{array}{l}\text { Bata de tela con manga larga } \\
\text { Bata de manga larga antifluido } \\
\text { o bata de tela con manga } \\
\text { larga y delantal plástico }\end{array}$ & $\checkmark$ & $\checkmark$ \\
\hline $\begin{array}{l}\text { Mascarilla quirúrgica } \\
\text { desechable }\end{array}$ & & \\
\hline $\begin{array}{l}\text { Tapabocas N-95 } \\
\text { Guantes de nitrilo o látex }\end{array}$ & $\checkmark$ & $\checkmark$ \\
\hline $\begin{array}{l}\text { Monogafas } \\
\text { Gorro }\end{array}$ & $\checkmark$ & $\checkmark$ \\
\hline $\begin{array}{l}\text { Careta o pantalla facial } \\
\text { completa }\end{array}$ & $\checkmark$ & $\checkmark$ \\
\hline
\end{tabular}

** Mascarilla quirúrgica desechable: también para áreas comunes sin contacto con pacientes. EPP: elementos de protección personal. Alto riesgo: PFP con generación de aerosoles.

\section{Recomendaciones para la fase luego de la pandemia}

Regrese al cumplimiento de los estándares preCOVID-19 para la prestación de servicios de función pulmonar, manteniendo los requisitos que garanticen servicios de buena calidad y seguridad de sus pacien- 
tes y del recurso humano. Resolución 00002003 del Ministerio de Salud y Protección Social de Colombia.

\section{Lecturas recomendadas}

- Guerra A, Torralba Y, Díaz D, Angulo M. Recomendaciones de prevención de infección por coronavirus en las unidades de función pulmonar de los diferentes ámbitos asistenciales. SEPAR, SEAIC, AET. 2020. doi: 10.13140/RG.2.2.12006.98884.

- Sociedad Uruguaya de Neumología [Internet]. Recomendaciones para realizar estudios funcionales respiratorios durante la epidemia causada por el coronavirus SARS-CoV-2 (COVID-19). Última actualización: marzo de 2020. Disponible en: https://suneumo.org/articulos-nacionales/recomendaciones-para-realizar- estudios-funcionales-respiratorios-durante-la-epidemia-causadapor-el-coronavirus-sars-cov-2-covid19\#attachmentsHeader

- McGowan A, Sylvester K, Burgos F, Boros P, De Jongh F, Kendrick A, et al. Recommendation from ERS Group 9.1 (Respiratory function technologists /Scientists) Lung function testing during COVID-19 pandemic and beyond We recommend the following safety measures for lung function testing based on the prevalence of COVID-19 in the community. 2020. doi: 10.13140/RG.2.2.28428.67207.

- $\quad$ Ministerio de Salud y Protección Social [Internet]. Orientaciones para la restauración gradual de los servicios de salud en las fases de mitigación y control de la emergencia sanitaria por COVID-19 en Colombia. Última visita: mayo de 2020. Disponible en: https:/www.minsalud.gov.co/Ministerio/Institucional/Procesos $\% 20 \mathrm{y} \% 20$ procedimientos/PSSS05.pdf 